\title{
YY FILTER - A PARADIGM OF DIGITAL SIGNAL PROCESSING
}

\author{
MASAAKI NAGAHARA
}

\begin{abstract}
YY filter, named after the founder Prof. Yutaka Yamamoto, is a digital filter designed by sampled-data control theory, which can optimize the analog performance of the signal processing system with $\mathrm{AD} / \mathrm{DA}$ converters. This article discusses problems in conventional signal processing and introduces advantages of the YY filter.
\end{abstract}

\section{INTRODUCTION}

YY filter is named after Prof. Yutaka Yamamoto, who is the founder of the modern sampled-data control theory. Before introducing the filter, I would like to write about him.

Prof. Yutaka Yamamoto has published a textbook on mathematics 21] in 1998. In that year, I was an undergraduate student in Kobe University, and I started studying control theory. I bought the book at that time, and found it very attractive. Affected by his book, I desired to be supervised by Prof. Yutaka Yamamoto in Kyoto University. I then luckily entered the university, and I began to study as a graduate student. I have studied sampled-data control and its application to digital signal processing. This study has started by Khargonekar and Yamamoto [6], which Prof. Yamamoto has been energetically addressing. Under his supervision, I finished my doctoral thesis titled "Multirate Digital Signal Processing via SampledData $H^{\infty}$ Optimization," 8 in 2003. This study has been of capital interest to me. I now begin the introduction of this study, YY filters.

In signal processing, signal reconstruction is a fundamental problem. For this problem, Shannon sampling theorem [14, 15] is widely used. This theorem is based on the assumption that the analog signal to be reconstructed is fully band-limited up to the Nyquist frequency. This assumption is however not realistic, since no real analog signals are fully band-limited. To such problems, sampled-data control theory has been applied in [6]. This is the first article of YY filter, which solves the delayed signal reconstruction problem. Based on this study, many researches have been made: multirate signal reconstruction 24], wavelet expansion [4] audio signal compression [1, fractional delay filters [10, image processing [3], adaptive filtering [11, probability density estimation [9], and repetitive control [12].

In this article, I omit discussion on these applications as space is limited, and I will concentrate on problems in Shannon's theorem (or its generalization) and advantages of the YY filter over the conventional theorem.

\section{Problems in Sampling Theorem}

2.1. Shannon sampling theorem. Let $x$ be a continuous-time signal in $L^{2}$, the Lebesgue spaces consisting of the square integrable real functions on $\mathbb{R}=$ 


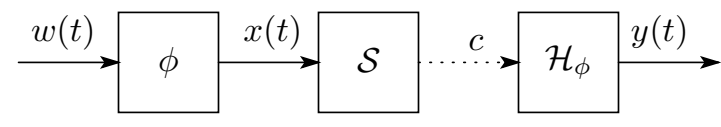

FiguRE 1. Shannon sampling theorem; the signal $w \in L^{2}$ is bandlimited by $\phi$ and sampled by the ideal sampler $\mathcal{S}$. Then an analog signal $y$ is produced by the hold device $\mathcal{H}_{\phi}$ to reconstruct $x$.

$(-\infty, \infty)$. The problem here is to recover the original signal $x$ from its sampled data $\{x(n h)\}_{n \in \mathbb{Z}}$, where $h>0$ is the sampling period. This problem is however ill-posed unless there is an a priori condition on the original signal $x$. The sampling theorem, usually attributed to Shannon, answers this question under the hypothesis of band-limited signals [14, 15]. That is, it is assumed that the support of the Fourier transform $\hat{x}(\mathrm{j} \omega)$ of $x$ is limited to the frequency range lower than the Nyquist frequency $\pi / h$ :

Theorem 1 (Whittaker-Shannon). Suppose that $x \in L^{2}$ is fully band-limited, i.e.,

$$
x \in B L^{2}:=\left\{x \in L^{2}: \hat{x}(\mathrm{j} \omega)=0,|\omega| \geq \pi / h\right\} .
$$

Then the following formula uniquely determines $x$ :

$$
x(t)=\sum_{n=-\infty}^{\infty} x(n h) \phi(t-n h), \quad t \in \mathbb{R},
$$

where $\phi(t):=\operatorname{sinc}(t / h):=\sin (\pi t / h) /(\pi t / h)$.

The reconstruction procedure is shown in Fig. 1. In this figure, the signal $w \in L^{2}$ is convoluted (or filtered) by $\phi$, i.e.,

$$
x(t)=\int_{-\infty}^{\infty} \phi(t-\tau) w(\tau) \mathrm{d} \tau=(\phi * w)(t) .
$$

Then the signal $x$ is in $B L^{2}$ (i.e., band-limited) since $\hat{\phi}(\mathrm{j} \omega)=1$ if $\omega \in(-\pi, \pi)$ and $\hat{\phi}(\mathrm{j} \omega)=0$ if $\omega \notin(-\pi, \pi)$. The signal $x$ is sampled by the ideal sampler $\mathcal{S}$ with the sampling period $h$ :

$$
(\mathcal{S} x)[n]:=x(n h), \quad n \in \mathbb{Z} .
$$

Then the discrete-time signal $c=\mathcal{S} x$ becomes an analog signal $y$ by the hold device $\mathcal{H}_{\phi}$ :

$$
\left(\mathcal{H}_{\phi} c\right)(t):=\sum_{n=-\infty}^{\infty} c[n] \phi(t-n h) .
$$

By the sampling theorem, the reconstructed signal $y$ is exactly equal to $x$ (not $w$ ).

Shannon sampling theorem is a beautiful result and is the fundamental theory for the conventional digital signal processing. However we can find the following questions in real applications:

- The band-limiting assumption (1) does not hold for real signals such as audio, image, or video signals.

- The reconstruction formula (2) is hard to implement on a real device, since the sinc function has infinite support, in particular it is not causal. 


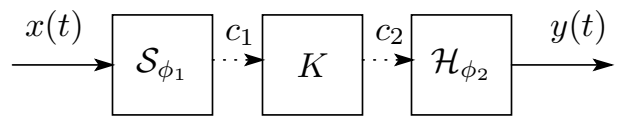

FiguRE 2. Generalized sampling theorem; the signal $x \in V\left(\phi_{2}\right)$ is sampled by the generalized sampler $\mathcal{S}_{\phi_{1}}$ to become the discretetime signal $c_{1}$. Then $c_{1}$ is filtered by $K$ to become $c_{2}$. Finally, an analog signal $y$ is produced by the hold device $\mathcal{H}_{\phi}$ to reconstruct $x$.

2.2. Generalized sampling theorem. The sampling theory mentioned above has been extended to more general case [17, 15], that is, the function $\phi$ is not necessarily a sinc function, and the sampler is a generalized sampler $\mathcal{S}_{\phi_{1}}$ defined by

$$
\left(\mathcal{S}_{\phi_{1}} x\right)[n]:=\int_{-\infty}^{\infty} \phi_{1}(n h-\tau) x(\tau) \mathrm{d} \tau=\left\langle x, \phi_{1}(\cdot-n h)\right\rangle, \quad n \in \mathbb{Z} .
$$

In this definition, we have $\mathcal{S}_{\phi_{1}} x=\mathcal{S}\left(\phi_{1} * x\right)$, and hence the function $\phi_{1}$ is considered as the impulse response of the acquisition device. Fig. 2 shows a generalized situation. In this figure, the analog input $x$ is sampled by the generalized sampler $\mathcal{S}_{\phi_{1}}$. Then the sampled signal $c_{1}$ is filtered by a digital filter $K$, and then an analog signal $y$ is obtained by the hold device $\mathcal{H}_{\phi_{2}}$. In this setting, a generalized sampling theorem is proposed by [17. The idea is the notion of consistency: the output $y$ in Fig. 2 can be perfectly reconstructed by the same system, that is, for all $n \in \mathbb{Z}$,

$$
\left\langle x, \phi_{1}(\cdot-n h)\right\rangle=\left\langle y, \phi_{1}(\cdot-n h)\right\rangle .
$$

This implies that the reconstruction system works as a projector. To achieve consistency, the optimal filter $K$ which is linear and time-invariant (LTI) is constructed by the oblique projection of $x$ onto $V\left(\phi_{2}\right)$ perpendicular to $V\left(\phi_{1}\right)$, where $V\left(\phi_{1}\right)$ and $V\left(\phi_{2}\right)$ are closed subspaces in $L^{2}$, which is defined by

$$
V\left(\phi_{i}\right):=\left\{x=\sum_{n=-\infty}^{\infty} c[n] \phi_{i}(t-n h), c \in \ell^{2}\right\}, \quad i=1,2 .
$$

By the oblique projection, the following generalized sampling theorem is obtained [17.

Theorem 2 (Unser and Aldroubi). Suppose that $x \in V\left(\phi_{2}\right)$ and the filter

$$
A_{12}(z)=\sum_{n=-\infty}^{\infty}\left\langle\phi_{1}(\cdot-n h), \phi_{2}\right\rangle z^{-n}
$$

is invertible1. Then the following formula uniquely determines $x$ :

$$
x(t)=\sum_{n=-\infty}^{\infty}\left(c_{1} * k\right)[n] \phi_{2}(t-n h), \quad t \in \mathbb{R}
$$

where $c_{1}:=\mathcal{S}_{\phi_{1}} x$ and $k$ is the impulse response of $K(z)=A_{12}(z)^{-1}$.

The assumption $x \in V\left(\phi_{2}\right)$ can be interpreted as a generalized band-limiting condition. Then we again have a problem of non-band-limited inputs, that is, $x \notin V\left(\phi_{2}\right)$. In this case, the reconstructed signal $y$ can have a large error [13.

\footnotetext{
${ }^{1}$ This means that $A_{12}(z)$ has no zeros on the unit circle in $\mathbb{C}$.
} 
Moreover, it is possible that the optimal filter will be unstable. This problem is discussed precisely in the next subsection.

2.3. Causality and stability. In real-time systems, causality is a necessary condition for signal processing. For the sake of simplicity, we assume 2 that $\phi_{1}(t)=$ $\phi_{2}(t)=0$ if $t<0$. Then the causality of the reconstruction system in Fig. 2 depends on the causality of the filter $K$. If the impulse response $\{k[n]\}$ of the filter $K$ satisfies $k[n]=0, n<0$, then the reconstruction system is causal. However, in many cases, the filter $K$ may be non-causal, for example, in the case of polynomial splines [18, and exponential splines [19, 16. This is because the filter $K(z)$ has poles outside of the unit circle in $\mathbb{C}[18,13$. In particular, it is shown 13 that high order exponential splines can produce filters with poles outside the unit circle provided that the sampling time is sufficiently small. Therefore, if the non-causal filter is realized as a causal one, the poles outside the unit circle lead to an unstable filter.

2.4. Summary. The problems in (generalized) sampling theorem discussed above are the following:

(1) If the input signal $x$ is not (generalized) band limited, the reconstructed signal can show a large error. In other words, the reconstruction is not robust against uncertainty of input signals.

(2) The reconstruction system can be non causal.

(3) The causal realization of the reconstruction filter $K$ can be unstable.

\section{Sampled-Data $H^{\infty}$ Optimal Signal Reconstruction - YY Filter}

As we see in the previous section, (generalized) sampling theorem has three problems: robustness, causality and stability. In this section, we introduce a new signal processing, sampled-data signal processing, or $Y Y$ filter, which is based on sampled-data control theory.

3.1. Problem formulation. The main reason to adopt sampled-data control theory is that we can design a digital filter which optimizes the intersample behavior. In other words, we can minimize the reconstruction error for non-band-limited signals. Moreover, we adopt the $H^{\infty}$ performance index for this optimization. By $H^{\infty}$ optimization, we can gain the robustness against the input uncertainty.

In the sampling theorem, the optimal reconstruction is a projector on a subspace in $L^{2}$, in which for every input the error is minimized in $L^{2}$ sense. This means that the error depends on the input and there can be an input for which the error can be arbitrary large. On the other hand, $H^{\infty}$ optimization is an optimization for the worst case, by which we can guarantee an error level for any inputs. This leads to the robustness against the input uncertainty.

It is obvious that there is no optimal filter $K$ which minimizes the error for all signals in $L^{2}$, or the optimal filter can be $K=0$. To reconstruct or interpolate the intersample data, we should assume some a priori information for the inputs. Therefore, we assume that the inputs are in the following subspace in $L^{2}$,

$$
F L^{2}:=\left\{F w: w \in L^{2}\left(\mathbb{R}_{+}\right)\right\}
$$

\footnotetext{
${ }^{2}$ If the assumption does not hold, the reconstruction system can be non-causal.
} 


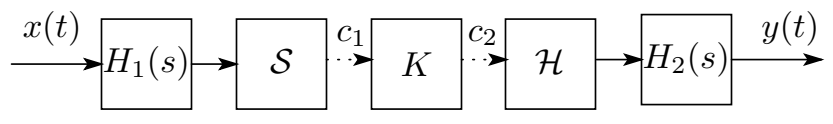

FiguRE 3. Signal Reconstruction; the signal $x \in L^{2}$ is filtered by an analog filter $H_{1}(s)$ and sampled by the ideal sampler $\mathcal{S}$. Then an analog signal $y$ is produced by the zero-order hold $\mathcal{H}$ and an analog filter $H_{2}(s)$.

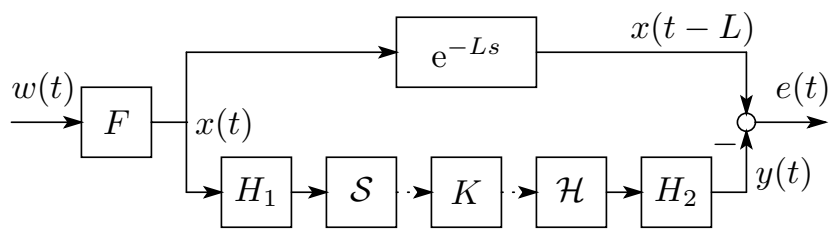

FiguRE 4. Error system $\mathcal{E}(K)$

where $F$ is an analog filter which is stable and strictly causal, and $L^{2}\left(\mathbb{R}_{+}\right)$is the Lebesgue spaces consisting of the square integrable real functions on $\mathbb{R}_{+}=[0, \infty)$. The filter $F$ is an analog model of the input signals. The space $L^{2}\left(\mathbb{R}_{+}\right)$is a subspace of $L^{2}$, by which we can take account of causality and stability of the reconstruction system. Our signal subspace $F L^{2}$ is in a sense larger than $B L^{2}$ or $V\left(\phi_{2}\right)$ because every signal in $B L^{2}$ or $V\left(\phi_{2}\right)$ can be expanded by $\{\operatorname{sinc}(t-n T)\}$ or $\left\{\phi_{2}(t-n T)\right\}$, on the other hand, $F L^{2}$ needs $\left\{\phi\left(2^{-m}(t-n T)\right)\right\}$ for some $\phi$ (wavelet expansion [20]). In other words, a signal in $F L^{2}$ can contain arbitrary high frequency components, the decay rate of which is governed by the filter $F$.

To optimize for the worst case, we consider the following performance index:

$$
J(K)=\sup _{\substack{x \in F L^{2} \\ x \neq 0}} \frac{\left\|\left(\mathrm{e}^{-L s}-H_{2} \mathcal{H} K \mathcal{S} H_{1}\right) x\right\|_{L^{2}\left(\mathbb{R}_{+}\right)}}{\|x\|_{L^{2}\left(\mathbb{R}_{+}\right)}} .
$$

This is equivalent to the $H^{\infty}$ norm of the sampled-data error system

$$
\mathcal{E}(K):=\left(\mathrm{e}^{-L s}-H_{2} \mathcal{H} K \mathcal{S} H_{1}\right) F .
$$

The block diagram of this error system is shown in Fig. 4.

3.2. Computation of YY filter. The optimal filter $K_{\text {opt }}$ which minimizes $J(K)$ in (3) can be obtained by numerical computation. To compute the optimal filter $K_{\text {opt }}$, we discretize the sampled-data error system $\mathcal{E}(K)$ in (4) by approximation [5, 23] or $H^{\infty}$ discretization [7. We here discuss the approximation technique for minimizing $J(K)$ in (3). We first introduce fast sampling and fast hold. Let $\mathcal{S}_{N}$ and $\mathcal{H}_{N}$ are respectively the ideal sampler and the zero-order hold with period $h / N$, where $N$ is a positive integer $(N \geq 2)$. Then the system $\mathcal{S}_{N} \mathcal{E}(K) \mathcal{H}_{N}$ becomes a discrete-time multi-rate system with sampling periods $h$ and $h / N$. Then we 


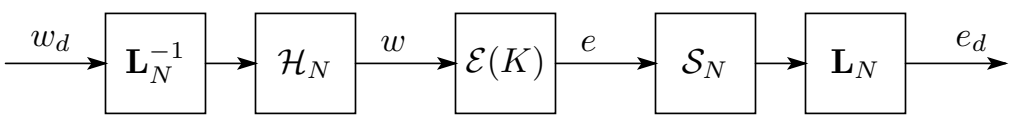

Figure 5. Fast discretization of the sampled-data system $\mathcal{E}(K)$ : $\mathbf{L}_{N}$ is the blocking operator, $\mathcal{S}_{N}$ and $\mathcal{H}_{N}$ are respectively the fast sampler and the fast hold with sampling period $h / N$.

introduce the blocking operator $\mathbf{L}_{N}$, or the discrete-time lifting operator [2, 8]:

$$
\mathbf{L}_{N}:\{v[0], v[1], v[2], \ldots\} \mapsto\left\{\left[\begin{array}{c}
v[0] \\
v[1] \\
\vdots \\
v[N-1]
\end{array}\right],\left[\begin{array}{c}
v[N] \\
v[N+1] \\
\vdots \\
v[2 N-1]
\end{array}\right], \ldots\right\} .
$$

This operator converts a 1-dimensional signal $v$ into an $N$-dimensional signal and the sampling rate becomes $N$ times slower. This operation makes it possible to equivalently convert multirate systems into single-rate ones, and hence the analysis and design become easier. By using this operator, the system $E_{N}(K)$ defined by

$$
E_{N}(K):=\mathbf{L}_{N} \mathcal{S}_{N} \mathcal{E}(K) \mathcal{H}_{N} \mathbf{L}_{N}^{-1}
$$

becomes a discrete-time LTI system. Moreover, we can say that for any integer $N \geq 2$ and any stable $K$, there exist discrete-time LTI systems $G_{1, N}, G_{2, N}$ and $G_{3, N}$ such that 8

$$
E_{N}(K)=G_{1, N}+G_{2, N} K G_{3, N},
$$

and the LTI system $E_{N}(K)$ is approximation of $\mathcal{E}(K)$ in the sense that 23]

$$
\lim _{N \rightarrow \infty}\left\|E_{N}(K)\right\|_{\infty} \rightarrow J(K)=\|\mathcal{E}(K)\|_{\infty}
$$

The optimization of minimizing $E_{N}(K)$ is easily done by using discrete-time $H^{\infty}$ optimization technique. We can therefore obtain a stable and causal filter $K$ which approximates the optimal filter $K_{\mathrm{opt}}$.

3.3. Robustness. Next let us consider robustness against uncertainty of the analog signal model $F(s)$. In practice, $F(s)$ cannot be identified exactly. We therefore partially circumvent this defect by discussing the robustness of the filter against uncertainty of $F(s)$. Let us assume the unstructured uncertainty of the following type:

$$
\begin{gathered}
F_{\Delta}(s):=F(s)(1+\Delta(s)), \quad \mathcal{E}^{\Delta}(K):=\left(\mathrm{e}^{-L s}-H_{2} \mathcal{H} K \mathcal{S} H_{1}\right) F_{\Delta}, \\
\Delta \in \Delta:=\left\{\Delta:\|1+\Delta\|_{\infty} \leq \gamma\right\} .
\end{gathered}
$$

Then we have the following proposition:

Proposition 1. For any stable $K$ and $\Delta \in \Delta$, we have $\left\|\mathcal{E}^{\Delta}(K)\right\|_{\infty} \leq \gamma\|\mathcal{E}(K)\|_{\infty}$.

By this proposition, the nominal performance $\|\mathcal{E}(K)\|_{\infty}$ is guaranteed against the perturbation $\Delta \in \Delta$ if $\gamma \leq 1$. In some cases, it is possible that $\gamma=1$, in which case the performance is bounded as illustrated in Fig. 6. This means that if we take $F(s)$ that covers all possible gain characteristics of the input analog signals, it gives a bound for the error norm. This at least partially justifies the choice of the first-order weighting $F(s)$ in the previous section. 


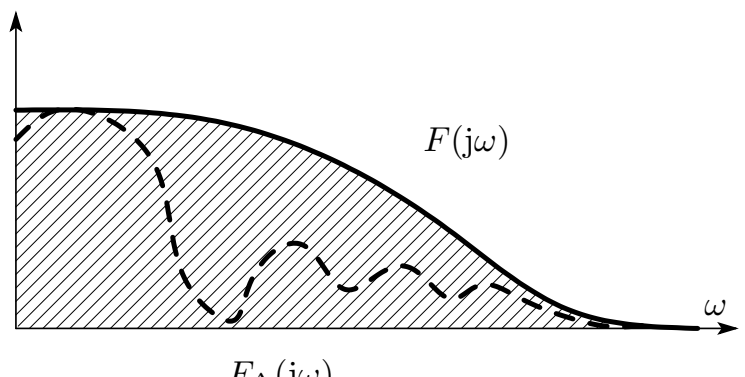

$F_{\Delta}(\mathrm{j} \omega)$

Figure 6. Nominal filter $F(s)$ (solid) and perturbed $F_{\Delta}(s)$ (dash)

3.4. FIR YY filter by LMI. The error system (4) or (5) is affine in the filter $K$ to be designed. By this fact, we can design the optimal FIR (finite impulse response) filter of the form

$$
K(z)=\sum_{n=0}^{N} a_{n} z^{-n}
$$

By this, the error system (5) is affine in the design parameter $a_{0}, a_{1}, \ldots, a_{N}$. It follows that the optimization of minimizing $\left\|E_{N}(K)\right\|_{\infty}$ can be described by an LMI (linear matrix inequality) by the bounded real lemma or Kalman-YakubovicPopov lemma 22. The optimization with an LMI can be solved easily by computer softwares.

3.5. Summary. The advantages of the YY filter discussed in this section are the following:

(1) the optimal filter is always causal and stable.

(2) the design takes the inter-sample behavior into account.

(3) the system is robust against the uncertainty of input signals.

(4) the optimal FIR filter is also obtainable via an LMI.

\section{Conclusions}

In this article, problems in Shannon's theorem have been pointed out and the advantages of YY filter over the conventional signal processing have been introduced. In fact, YY filters are implemented in commercial MD players, silicon-audio devices, and mobile phones. One of future works is design of adaptive YY filters.

\section{REFERENCES}

[1] Ashida, S., Kakemizu, H., Nagahara, M., Yamamoto, Y.: Sampled-data audio signal compression with Huffman coding. In: SICE Annual Conf., pp. 972-976 (2004)

[2] Chen, T., Francis, B.A.: Optimal Sampled-data Control Systems. Springer (1995)

[3] Kakemizu, H., Nagahara, M., Kobayashi, A., Yamamoto, Y.: Noise reduction of JPEG images by sampled-data $H^{\infty}$ optimal $\varepsilon$ filters. In: SICE Annual Conf., pp. 1080-1085 (2005)

[4] Kashima, K., Yamamoto, Y., Nagahara, M.: Optimal wavelet expansion via sampled-data control theory. IEEE Signal Process. Lett. 11, 79-82 (2004)

[5] Keller, J.P., Anderson, B.D.O.: A new approach to the discretization of continuous-time controllers. IEEE Trans. Autom. Control 37, 214-223 (1992)

[6] Khargonekar, P.P., Yamamoto, Y.: Delayed signal reconstruction using sampled-data control. In: IEEE CDC, pp. 1259-1263 (1996)

[7] Mirkin, L., Tadmor, G.: Yet another $H^{\infty}$ discretization. IEEE Trans. Autom. Control 48, 891-894 (2003) 
[8] Nagahara, M.: Multirate Digital Signal Processing via Sampled-Data $H^{\infty}$ Optimization. Ph.D thesis, Kyoto University (2003)

[9] Nagahara, M., Sato, K.I., Yamamoto, Y.: $H^{\infty}$ optimal nonparametric density estimation from quantized samples. In: ISCIE SSS (2008)

[10] Nagahara, M., Yamamoto, Y.: Optimal design of fractional delay filters. In: IEEE CDC, pp. 6539-6544 (2003)

[11] Nagahara, M., Yamamoto, Y.: Hybrid design of filtered- $x$ adaptive algorithm via sampleddata control theory. In: IEEE ICASSP, pp. 353-356 (2008)

[12] Nagahara, M., Yamamoto, Y.: Robust repetitive control by sampled-data $H^{\infty}$ filters. In: IEEE CDC (2009)

[13] Nagahara, M., Yamamoto, Y., Khargonekar, P.P.: Stability of signal reconstruction filters via exponential splines. In: 17th IFAC World Congress, pp. 1414-1419 (2008)

[14] Shannon, C.E.: Communication in the presence of noise. Proc. IRE 37(1), 10-21 (1949)

[15] Unser, M.: Sampling - 50 years after Shannon. Proc. IEEE 88(4), 569-587 (2000)

[16] Unser, M.: Cardinal exponential splines: Part II — Think analog, act digital. IEEE Trans. Signal Process. 53(4), 1439-1449 (2005)

[17] Unser, M., Aldroubi, A.: A general sampling theory for nonideal acquisition devices. IEEE Trans. Signal Process. 42(11), 2915-2925 (1994)

[18] Unser, M., Aldroubi, A., Eden, M.: B-spline signal processing: Part II — Efficient design and applications. IEEE Trans. Signal Process. 41(2), 834-848 (1993)

[19] Unser, M., Blu, T.: Cardinal exponential splines: Part I - Theory and filtering algorithms. IEEE Trans. Signal Process. 53(4), 1425-1438 (2005)

[20] Vetterli, M., Kovaçević, J.: Wavelets and Subband Coding. Prentice Hall (1995)

[21] Yamamoto, Y.: Mathematics for Systems and Control. Asakura (1998)

[22] Yamamoto, Y., Anderson, B.D.O., Nagahara, M., Koyanagi, Y.: Optimizing FIR approximation for discrete-time IIR filters. IEEE Signal Process. Lett. 10(9), 273-276 (2003)

[23] Yamamoto, Y., Madievski, A.G., Anderson, B.D.O.: Approximation of frequency response for sampled-data control systems. Automatica 35(4), 729-734 (1999)

[24] Yamamoto, Y., Nagahara, M., Fujioka, H.: Multirate signal reconstruction and filter design via sampled-data $H^{\infty}$ control. In: MTNS2000 (2000)

Masaaki Nagahara is with Graduate School of Informatics, Kyoto University, KyOTO 606-8501, JAPAN (NAGAHARA@IEEE.ORG). 